\title{
En el camino: Misioneros, recorridos y paisajes (Esteco, s. XVI- XVII; Tierra del Fuego, s. XIX-XX)
}

\section{On the way: Missionaries, routes and landscapes Esteco $\left(16^{\text {th }}\right.$ - $18^{\text {th }}$ centuries $)$ and Tierra del Fuego $\left(19^{\text {th }}-20^{\text {th }}\right.$ centuries $)$}

\author{
Enviado em: 04/06/2017 \\ Aceito em: 04/06/2017 \\ SALERNO, Melisa A. ${ }^{1}$ \\ MARSCHOFF, María. ${ }^{2}$
}

\begin{abstract}
Resumen:
La acción misional tradicionalmente supuso anunciar el Evangelio entre aquéllos que lo desconocían. Nuestro trabajo considera dos casos de estudio que involucraron experiencias misionales, temporal y espacialmente diferentes. La primera de ellas correspondió con la labor de los jesuitas en Esteco, entre los siglos XVI y XVII; y la segunda, con la obra de los salesianos en Tierra del Fuego, hacia fines del siglo XIX y principios del XX. En este trabajo consideramos la dimensión espacial de la acción misional, atendiendo al vínculo entre los religiosos y los paisajes. En particular, enfatizamos la importancia de abordar un paisaje vivido y recorrido, caracterizado por su dinámica y mutabilidad. Nuestro objetivo consiste en analizar "paisajes en movimiento", discutiendo la diversidad de recorridos efectuados, la materialidad de los paisajes físicos y habitados, y las prácticas y experiencias de los religiosos que los recorrieron. Los resultados no sólo permiten atender a ciertas peculiaridades de los casos de estudio, sino también debatir el posible impacto de integrar los recorridos en los estudios sobre paisajes y el mundo colonial en arqueología.
\end{abstract}

Palabras clave: misioneros, recorridos, Tierra del Fuego.

\footnotetext{
${ }^{1}$ IMHICIHU-CONICET

${ }^{2}$ IDH-CONICET
} 


\title{
Resumo:
}

A ação missionária tradicionalmente envolveu a proclamação do Evangelho entre aqueles que 0 desconheciam. Nosso trabalho considera dois estudos de caso envolvendo experiências missionárias, temporal e espacialmente diferentes. A primeira correspondeu à obra dos jesuítas em Esteco, entre os séculos XVI e XVII; e a segunda, á obra dos salesianos na Tierra del Fuego, no final do século XIX e no início do XX. Neste trabalho consideramos a dimensão espacial da ação missionária, considerando a relação entre os religiosos $e$ as paisagens. Em particular, destaca-se a importância de estudar paisagens vividas e percorridas, caracterizadas pela sua dinâmica e mutabilidade. Nosso objetivo é analisar os "paisagens em movimento", discutindo a diversidade de caminhos seguidos, a materialidade das paisagens físicas e habitadas, e as práticas e experiências dos sacerdotes. Os resultados servem não somente para discutir certas particularidades dos estudos de caso, mas também para considerar o possível impacto da integração do movimento em o estudo das paisagens e o mundo colonial em arqueologia.

Palavras-chave: missionários, caminhos, Tierra del Fuego.

\begin{abstract}
Christian missionary activity has typically entailed the proclamation of the Gospel to those had previously been unaware of it. This article examines two case studies of Christian missionary expeditions from differing historical and geographical contexts. The first case study is of Jesuits in Esteco en the $16^{\text {th }}$ and $17^{\text {th }}$ centuries, and the second case study is of Salesian missionaries in Tierra del Fuego in the late $19^{\text {th }}$ and early $20^{\text {th }}$ centuries. In this article, we examine spatial aspects of missionary activity, taking into account the relationship of the missionaries to the landscape. The landscapes, which are traversed and interacted with, characterized by their changeable dynamics, are particularly taken into account. Our objectiveis to analyze "landscapes in movement"examining the different paths taken, thematerial aspects of the inhabited landscapes, as well as the practices and experiences of the priests. The results of this study are relevant not only to examine these two case studies, but also to consider the impact
\end{abstract}


that movement has on thestudy of landscapes and the colonial period within the archaeological context.

Key words: missionaries, routes, Tierra del Fuego.

\section{Introducción}

Recientemente, las autoras de este trabajo comenzamos a estudiar las experiencias de las personas en dos contextos espacial y temporalmente diferentes del extremo sur de Sudamérica. Desde hacía algunos años, María Marschoff analizaba diversos aspectos de las relaciones coloniales en la región aproximadamente coincidente con la jurisdicción de la ciudad de Esteco, perteneciente a la gobernación española del Tucumán (norte del actual territorio de Argentina), entre los siglos XVI y XVII (MARSCHOFF et al., 2014; PORTERIE \& MARSCHOFF, 2014). Mientras tanto, en el marco de un equipo interdisciplinario, Melisa A. Salerno hacía algo semejante en la región de Tierra del Fuego (sur de Argentina y Chile), entre fines del siglo XIX y la primera mitad del XX (GUICHÓN et al., 2014; SALERNO \& GUICHÓN, 2015). En ambos casos, misioneros pertenecientes a diferentes órdenes buscaron evangelizar a los indígenas. Mientras en Esteco este rol fue desempeñado por los jesuitas; en Tierra del Fuego, fue protagonizado por los salesianos.

Las características de ambos casos hacían posible el diálogo. El período considerado para Esteco y Tierra del Fuego comprendía la llegada de los religiosos, así como el desarrollo y declive de la acción evangelizadora en cada una de las regiones. Si bien Esteco se enmarcaba en el período colonial español, y Tierra del Fuego en el de consolidación de los estados nacionales de Argentina y Chile, ambos casos formaban parte de un proceso de colonización extendido. Aunque los escenarios presentaban escalas espaciales diferentes, ambos permitían abordar las redes de acción de grupos bien definidos de misioneros. Si bien el conocimiento y la presencia colonial previos eran particulares, tanto Esteco como Tierra del Fuego comprometían un mosaico de territorios total y/o parcialmente explorados e inexplorados. Asimismo, en ambos casos, los indígenas que constituían el centro de la acción misional eran nómades. 
Pensando en abordar la acción misional, una de las primeras cosas que creímos oportuno atender fue su dimensión espacial. Algunos antecedentes sobre la temática enfatizaban las unidades administrativas definidas por el derecho canónico. Las notas sobre Esteco mencionaban la Provincia Jesuítica del Paraguay; y las de Tierra del Fuego, la Prefectura Apostólica de la Patagonia Meridional, Magallanes, Tierra del Fuego e Islas Malvinas, desplegando mapas, precisando límites, definiendo extensiones. Otras referencias focalizaban en puntos concretos del espacio. Por lo general, en el caso de Esteco se consideraba la propia ciudad (dentro de una red comercial más amplia), y en menor medida las reducciones de San Francisco Javier y Miraflores (LOZANO, [1733] 1941; TORRE REVELLO, 1943; TOMASINI \& ALONSO, 2001; TOMASINI 2006); en Tierra del Fuego, por su parte, se insistía sobre la ciudad de Puntarenas, y las misiones de San Rafael y La Candelaria (CASALI, 2013; FERNÁNDEZ, 2014), brindando datos sobre su fundación, cantidad de indígenas, actividades cotidianas, entre otros (ALIAGA ROJAS, 2000; MARTINIC, 2011).

Inicialmente, comenzamos por consultar algunos documentos que permitían conocer de manera más o menos directa la acción de los religiosos en el espacio. Además de revisar diarios y crónicas, en el caso de Esteco recurrimos adicionalmente a las Cartas Anuas de la Provincia Jesuítica del Paraguay (un informe enviado por el Provincial al Padre General de la Compañía, donde se describían los principales sucesos ocurridos en el territorio en uno o más años); y en el de la Tierra del Fuego, al Boletín Salesiano (una publicación oficial de la orden, donde mensualmente se informaba sobre la situación de los misioneros en el globo, incluyendo Patagonia Austral). El relevamiento arrojó datos interesantes; especialmente, por el número y diversidad de referencias a exploraciones, viajes, visitas, senderos, derroteros y trayectos.

En ocasiones, las referencias a las unidades administrativas pueden comprometer un espacio abstracto, una suerte de "contenedor" (sensu THOMAS, 1994) que no siempre permite dar cuenta de las prácticas y experiencias de los religiosos. Mientras tanto, las referencias a puntos concretos, como ciudades, misiones y reducciones, pueden operar recortes sobre un espacio más extenso, fijando la acción de los religiosos e interrumpiendo sus flujos con el mundo. Los recorridos referidos por los documentos nos sumergieron en un paisaje en movimiento, 
un tanto diferente de aquél propuesto por algunos antecedentes. Teniendo en cuenta esta idea, comenzamos a pensar que los recorridos podían proporcionar una vía alternativa para abordar la relación entre los misioneros y el paisaje (sin necesidad de negar o sustituir otras aproximaciones).

Atender a los recorridos es más que marcar y conectar puntos fijos en el espacio. Considerar los recorridos supone aproximarnos a la relación entre quienes recorren el paisaje y el paisaje por ellos recorrido. Es a través de este vínculo que se modelan "paisajes en movimiento", caracterizados por su mutabilidad y dinamismo. En este marco, los recorridos no sólo se encuentran definidos por quienes recorren. También el paisaje recorrido impone sus posibilidades y límites al movimiento (TILLEY, 1994). La materialidad del paisaje recorrido puede suponer o no caminos construidos, capaces de dejar huellas a nivel arqueológico. Lo que aquí -sin embargoqueremos destacar es que esa materialidad simultáneamente involucra un paisaje físico (caracterizado por accidentes geográficos, flora, fauna, clima, caminos, edificios, ciudades, etc.) y habitado (caracterizado por la presencia de gente que, siendo copresente, también efectúa sus propios movimientos) (INGOLD, 2011).

El paisaje recorrido no es el mismo para todas las personas. Distintos grupos humanos responden a prácticas particulares, culturalmente informadas, sobre qué espacios recorrer y de qué maneras hacerlo (INGOLD \& LEE VERGUNST, 2008). Estas prácticas comprometen la materialidad del cuerpo propio, y se vinculan con experiencias que no son sólo kinestésicas (por involucrar el movimiento), sino especialmente sinestésicas (al suponer un entramado perceptual y emocional que excede las distinciones de los cinco sentidos aristotélicos). Los recorridos y las formas de recorrer se alimentan de encuentros previos con el espacio (ya sean propios o ajenos), siendo capaces de modelar otros encuentros de forma novedosa. Además, las prácticas y experiencias asociadas al recorrer son producidas por (a la vez que producen) una pluralidad de entendimientos prácticos sobre el espacio físico, el espacio habitado y quienes recorren.

Sintetizando, en este trabajo proponemos discutir la relación entre los misioneros y el paisaje, efectuando una primera aproximación a los paisajes en movimiento para los casos de Esteco y la Tierra del Fuego. Si bien no queremos descuidar el carácter dinámico de los vínculos, con el propósito de simplificar la 
presentación, en primer lugar describiremos la diversidad de recorridos efectuados. Posteriormente, abordaremos la materialidad de los espacios físicos y habitados, así como las prácticas y experiencias que caracterizaron a los misioneros como sujetos en movimiento.

Antes de pasar al análisis, quisiéramos agregar que las referencias que los documentos brindan sobre los recorridos en cada contexto son diferentes. Las descripciones para Esteco son mucho más escuetas. Por lo general, los recorridos son presentados como trayectorias; esto es, como enumeraciones de sitios por los que se pasa. Las referencias sobre el espacio físico son mínimas, habiendo mayor interés por el espacio habitado y el misionero como sujeto en movimiento. Los registros sobre Tierra del Fuego resultan contrastantes, brindando descripciones igualmente abundantes, detalladas y vívidas sobre los diferentes aspectos de los paisajes en movimiento. Los motivos de estas diferencias (incluyendo las formas de entender y narrar la experiencia en cada uno de los contextos) no son motivo de análisis en el presente trabajo, pero sin duda recibirán atención en el futuro.

\section{Recorridos entre los jesuitas y los salesianos}

En 1584, y respondiendo al llamado del gobernador y el obispo de la región, los jesuitas llegaron desde Lima a Tucumán. Entre ellos vino el padre Alonso Barzana, usualmente conocido como "el apóstol del Perú y Tucumán", quien inició junto con otros la exploración del territorio con fines evangelizadores (DEL TECHO, [1673] 1897; LOZANO [1745] 2010). Durante este primer período, algunos padres permanecieron en las ciudades españolas y fundaron casas. Sin embargo, la mayoría recorrió los caminos, visitando los lugares que habitaban los "indios de servicio" (DEL TECHO, [1673] 1897; LOZANO, [1733] 1941; GUEVARA, [1764] 1882). Hacia 1600, esta situación de "nomadismo" se puso en cuestión dentro de la propia orden (DEL TECHO, [1673] 1897). Así, en 1604 se creó la Provincia Jesuítica del Paraguay y se comenzaron a organizar residencias fijas. 
En 1617, los jesuitas se establecieron en la propia ciudad de Esteco, desde donde se irradió gran parte de la acción misional en la jurisdicción (DE OÑATE [1618] en RAVIGNANI \& LEONHART, 1929). En 1635, abandonaron el lugar en un escenario de guerra, hambre, pestes y un temblor (DE BEROA [1634] en MAEDER, 1990; DEL TECHO, [1673] 1897). Tan sólo regresaron a la región entre finales del siglo XVII y principios del siglo XVIII, durante dos lapsos breves en los que fundaron las misiones de San Francisco Javier y Miraflores respectivamente (MACHONI 1732; LOZANO, [1733] 1941). Siendo Esteco parte de la frontera con el "indígena hostil del Chaco" (LOZANO, [1733] 1941), el rol de los jesuitas implicó mediar entre estos grupos y los españoles. Por lo que describen las fuentes, en estas reducciones se habría intentado replicar el modelo de las misiones guaraníticas. La primera se abandonó por decisión del gobernador; y la segunda, por recrudecimiento de las hostilidades con los indígenas no-reducidos (LOZANO, [1733] 1941).

Los salesianos llegaron a Tierra del Fuego desde Italia, como parte del proyecto de Don Bosco de evangelizar la Patagonia (BRUNO, 1992; NICOLETTI, 2006). En 1883, la Santa Sede estableció la Prefectura Apostólica de Patagonia Meridional, Magallanes, Tierra del Fuego e Islas Malvinas; pero fue tan sólo cuatro años más tarde que se iniciaron acciones concretas sobre Tierra del Fuego. En ese entonces, Giuseppe Fagnano instaló la Casa Central en Puntarenas (el mayor poblado del área). Si bien existían algunos datos sobre Tierra del Fuego, gran parte de la región aún permanecía inexplorada. Así Fagnano junto con otros recorrió el archipiélago y la Isla Grande en sucesivos viajes. Finalmente, en 1889 se estableció la misión de San Rafael en Isla Dawson; y en 1893, la misión de Nuestra Señora de La Candelaria en Río Grande (ALIAGA ROJAS, 2000; CASALI, 2013).

La presencia de las misiones buscó reducir a los indígenas. Sin embargo, también inauguró una nueva fase de movimientos. La escasez de personal obligó a los religiosos a trasladarse periódicamente, de forma de atender las urgencias de las diversas casas. Además, la falta de autosuficiencia de los enclaves obligó periódicamente a transportar recursos (sobre todo, desde Puntarenas). El número de indígenas reducidos disminuyó de manera significativa con el paso del tiempo (NICOLETTI, 2006; CASALI, 2013). Ocasionalmente, los religiosos efectuaron "misiones" por el archipiélago y la Isla Grande. Las mismas se incrementaron, hasta 
que hacia 1910, se establecieron nuevas casas en Río Fuego y Lago Fagnano (BASCOPÉ JULIO, 2010). Las mismas -no obstante- no pudieron detener el declive de la acción evangelizadora en Tierra del Fuego.

\section{El paisaje recorrido en Esteco y Tierra del Fuego}

\section{El espacio físico}

En el caso de Esteco, las primeras exploraciones efectuadas por los jesuitas siguieron el curso de los ríos, aprovechando la presencia de población a lo largo de los mismos. Sin embargo, por lo general, los recorridos misionales utilizaron la red de caminos coloniales que unían distintas ciudades. Estos caminos, y por ende también los recorridos de los jesuitas, evitaban uno de los pocos rasgos físicos reiteradamente mencionados por las fuentes: el bosque o la "selva". La vegetación densa de monte, típica del Chaco, resultaba intransitable en muchos casos (especialmente, a caballo). Allí "se ocultaban", desde allí "salían" o hacia allí "huían" los indígenas (incluyendo los denominados "hostiles"). En este escenario, el bosque se transformó en un lugar "cerrado" para los españoles, una "barrera" infranqueable, una masa que incluso "invadía" los espacios de las ciudades.

En Tierra del Fuego, la mayor parte de los recorridos se efectuó por mar. En el caso de San Rafael, la navegación resultó inevitable, pues la misión se hallaba localizada en una isla. Los recorridos por mar que integraron Puntarenas y La Candelaria buscaron minimizar las incomodidades de la vía terrestre. La densidad de los bosques, sumada a la presencia de ríos y pantanos, hizo que los religiosos evitaran transitar el sur; especialmente, hasta que comenzaron a desarrollar misiones en busca de los indígenas replegados. La sección norte de la estepa se encontraba ocupada por campamentos mineros, y el área central por grandes estancias. Los recorridos terrestres que atravesaron estos paisajes fueron escasos, y comúnmente tuvieron lugar cuando no se disponía de comunicación por barco. 


\section{El espacio habitado}

En ambos casos, la acción misional se encontró focalizada en los indígenas. En la jurisdicción de Esteco los jesuitas se centraron inicialmente en los grupos que se hallaban encomendados. Este sistema de explotación forzaba a los nativos a vivir en función de las actividades productivas de los españoles. Asimismo, suponía graves dificultades para la evangelización: no sólo obligaba a los religiosos a buscar indígenas por diversas fincas; también incrementaba el potencial de "fuga". Hacia fines del siglo XVII y principios del XVIII, los jesuitas adoptaron un rol mediador con tribus "hostiles", no encomendadas, que habitaban por fuera de la jurisdicción de las ciudades y con quienes ensayaron reducciones, esperando que redujeran su movilidad voluntariamente y alcanzaran su conversión.

Por su parte, Tierra del Fuego era habitada por indígenas que luchaban por mantener su estilo de vida nómade, en un contexto que comenzaba a sentir la presencia de colonos. El conflicto que los mineros y estancieros mantuvieron con los indígenas (manifestado en la persecución e incluso la caza de nativos) fue funcional al establecimiento de misiones, ya que los indígenas acudían a estos lugares en busca de alimentos y protección. Asimismo, en algunas ocasiones, los indígenas eran entregados por los mismos estancieros o la policía (NICOLETTI, 2006; CASALI, 2013). Sin embargo, en cuanto la permanencia era presuntamente "voluntaria", no todos los indígenas estuvieron dispuestos a renunciar a su movilidad. Así, como resultado de éstas y otras circunstancias, los salesianos intentaron traerlos de regreso, 0 evangelizarlos en misiones volantes por los sitios que frecuentaban.

\section{Los misioneros como sujetos en movimiento}

Tanto en Esteco como en Tierra del Fuego, los documentos señalan la multiplicidad y asiduidad de los movimientos de los misioneros. Los caminos se recorrían a pie (cuando el terreno era accidentado), a caballo (siempre que fuera posible), e incluso en barco (en el caso de Tierra del Fuego). Los viajes podían durar 
días, semanas e incluso meses. Los relatos interesados por la experiencia cotidiana de los recorridos destacan el esfuerzo físico, los peligros y las privaciones enfrentadas. Esto dio lugar a la construcción de la imagen ideal del misionero: una persona sacrificada y resistente que podía incluso despreciar su propia vida en pos de su objetivo. Específicamente, misionar fue entendido como una vocación de origen divino: una "sed" o "fuego" que impulsaba a los miembros de las órdenes a recorrer caminos inhóspitos con el propósito de recolectar almas para el Señor.

\section{Conclusiones}

"Dejarnos llevar por los caminos" nos permitió construir un abordaje alternativo a la dimensión espacial de la acción misional. Tanto en Esteco como en Tierra del Fuego, los "paisajes en movimiento" de esa actividad involucraron el vínculo entre los religiosos que recorrieron, y una serie de espacios recorridos, simultáneamente físicos y habitados por otros que también recorrían. Fue en el desarrollo de su labor evangelizadora que los jesuitas y salesianos modelaron una red cambiante de recorridos, donde los rasgos físicos del paisaje, los indígenas y otros habitantes les impusieron posibilidades y obstáculos para la marcha. En este marco, el espacio no constituye un objeto pasivo sobre el que únicamente se impone un sujeto activo. El movimiento "une" y "dinamiza", de forma tal que los elementos vinculados se vuelven indisociables. Este tipo de reflexiones enfrenta las categorías fijas y contrapuestas, tradicionalmente naturalizadas por el pensamiento científico.

Si bien en el presente trabajo efectuamos una serie de escisiones, ellas sólo tuvieron un carácter analítico. Sin lugar a dudas, uno de nuestros principales desafíos en el futuro debe ser explorar nuevas metodologías y formas de escritura que permitan captar de manera adecuada el fluir de los movimientos. En relación a las categorías, quisiéramos efectuar una aclaración adicional. Al hablar de paisajes en movimiento no proponemos crear una nueva dicotomía entre movimiento y estaticidad. Desde nuestra posición, los recorridos suponen paradas y estadías, que simultáneamente pueden ser tan transitorias y transitadas como los propios caminos. Hablar de paisajes en movimiento pretende poner en foco una dimensión dinámica del paisaje, que quizás 
haya sido subestimada. Es tan sólo un calificativo que, desarrollada y consolidada nuestra propuesta, probablemente pueda ser descartado.

Tradicionalmente, la experiencia del recorrido entre los misioneros de Esteco y Tierra del Fuego no fue suficientemente considerada. Esta orientación no fue resultado exclusivo de "sesgos" presentes en las fuentes. Quizás los investigadores pusieron demasiado énfasis en el proyecto sedentarizador de los misioneros; esto es, en el deseo de reducir a los indígenas. En un mundo colonial de encuentros con grupos nómades, el sedentarismo fue discursivamente presentado como clave de la diferencia cultural y camino de civilización. Sin embargo, el proyecto sedentario no sólo encontró trabas entre los indígenas. Tampoco pudo ser alcanzado por los propios religiosos que, siendo pocos en número, debieron actuar sobre territorios vastos, con dificultades para la autosuficiencia en los enclaves. Probablemente, este mundo colonial necesitó mucho más movimiento que lo tradicionalmente pensado, obligándonos a repensar los discursos del colonialismo y sus contradicciones. Quizás nosotros, también, estemos envueltos en esos discursos, negando nuestros recorridos, y proyectando esa negación al pasado.

\section{Agradecimientos}

A Ricardo Guichón, Andrés Zarankin, Alfredo Tomasini, Beatriz Bixio, Ana Paula Porterie y Julia Simioli. A CONICET por el apoyo prestado. A Fernanda Codevilla Soares por la traducción al portugués del resumen. A la Organización de la II Semana de Arqueología de UNICAMP. Las investigaciones en Tierra del Fuego cuentan con financiamiento del Préstamo BID-PICT 0575.

\section{Referencias}

ALIAGA ROJAS, F. La misión salesiana en Isla Dawson (18891911). Santiago de Chile: Editorial Don Bosco, 2000.

BASCOPÉ JULIO, J. Documentos inéditos para la historia de Magallanes. Desvíos salesianos. La expedición de 1906 y los misioneros volantes. In: Magallania, Punta Arenas: vol. 38, $\mathrm{n}^{\circ} 2$, 2010. pp.249-259. 
BRUNO, C. La evangelización de la Patagonia y de la Tierra del Fuego. Rosario: Didascalia, 1992.

CASALI, R. Conquistando el fin del mundo. La misión La Candelaria y la salud de la población selk'nam (Tierra del Fuego, 1895-1931). Rosario: Prohistoria, 2013.

FERNÁNDEZ, A. Nota preliminar. En: Con letra de mujer: la crónica de las Hijas de María Auxiliadora en la Misión Nuestra Señora de la Candelaria, Tierra del Fuego. Buenos Aires: Didascalia, 2014.

GUICHÓN, R.; CASALI, R.; GARCÍA L., P.; SALERNO, M. \& GUICHÓN, R. Double coloniality in Tierra del Fuego, Argentina: A bioarchaeological and historiographical approach to Selk'nam demographics and health (La Candelaria mission, late $19^{\text {th }}$ and early $20^{\text {th }}$ centuries). In: MURPHY, M. \& KLAUS, $H$. (Eds.), Bioarchaeology of contact, colonial encounters and colonialism. Utah: Utah Valley University, 2014. En prensa.

INGOLD, T. Being alive: Essays on movement, knowledge and description. London: Routledge, 2011.

INGOLD, T. \& LEE VERGUNST, J. Introduction. In: INGOLD, T. \& LEE VERGUNST, J. (Eds.). Ways of walking: Ethnography and practice on foot. Ashgate: Aldershot, 2008.

MARSCHOFF, M., C. CASTIÑEIRA LATORRE \& SIMIOLI, J. Arqueoestratigrafía de referencia para el registro de la ocupación humana en el sitio de Esteco I (Departamento de Anta, Salta, Argentina) durante los siglos XVI y XVII. In: Chungara. Revista de Antropología Chilena, Arica: vol. 46, n 3, 2014. pp. 355-374.

NICOLETTI, M. Los misioneros salesianos y la polémica sobre la extinción de los selk'nam de Tierra del Fuego. In: Anthropologica, año 24, n²4, 2006. pp. 153-177.

ORELLANA, M. La misión salesiana en Isla Dawson: Intento por evitar la extinción fueguina. In: Revista Marina, Viña del Mar: $n^{\circ} 2$, 2011. pp. 181-187

PORTERIE, A. \& MARSCHOFF, M. Las experiencias de habitar un nuevo emplazamiento en Nuestra Señora de Talavera de Madrid (1609-1692). In: Ponencia presentada en el VI Congreso Iberoamericano de Ambiente y Calidad de Vida-7mo Congreso de Ambiente y Calidad de Vida. Facultad de Ciencias Exactas y Naturales, UNC: Catamarca, 2014.

SALERNO, M. \& GUICHÓN, R. Sobre la memoria y el olvido. Los difuntos selk'nam y el cementerio de la misión salesiana Nuestra Señora de La Candelaria (Río Grande, Tierra del Fuego). In: Magallania, Punta Arenas, 2015. Enviado. 
THOMAS, J. The politics of vision and the archaeologies of landscape. In: BENDER, B. (Ed.). Landscapes: Politics and perspectives. Oxford: Berg, 1993. pp. 19-48.

TILLEY,C. The phenomenology of landscape. Oxford: Berg, 1994.

TOMASINI, J.A. \& ALONSO, R.N. Esteco, el viejo. Breve historia y localización de Nuestra Señora de Talavera 1566-1609. Salta: Gráfica Editora, 2001.

TOMASINI, J.A. Los caminos desde Santiago del Estero hasta "Las Juntas" durante el período colonial (siglos XVI-XVIII). In: Revista Junta de Estudios Históricos del Chaco 3, Resistencia, 2006.

TORRE REVELLO, J. Esteco y Concepción del Bermejo: Dos ciudades desaparecidas. In: Publicaciones del Instituto de Investigaciones Históricas, Buenos Aires: n 85, 1943.

\section{Fuentes consultadas}

BARZANA, A. de. Carta de P. Alonso de Barzana, de la Compañía de Jesús, al P. Juan Sebastián, su provincial. En: BERBERIAN (Comp.), Cronistas del Tucumán, siglo XVI. Comechingonia. Revista de Antropología e Historia, Córdoba: p. 251-263, 1987 [1594].

BEROA, D. de. Anales de la provincia del Paraguay, desde el año de 32 hasta el de 34. In: MAEDER, E (Comp.). Cartas Anuas de la provincia jesuítica del Paraguay (1632 a 1634). Edición en Homenaje al Quinto Centenario del Descubrimiento de América, p. 23-51. Buenos Aires: Academia Nacional de la Historia, [1635] 1990.

Crónica del Padre Zenone sobre la Misión Nuestra Señora de La Candelaria (Inédito). Disponible en Museo Histórico y Natural Monseñor Fagnano (La Candelaria, Río Grande, Tierra del Fuego), [1898-1902].

Crónica de las Hermanas de María Auxiliadora sobre la Misión Nuestra Señora de La Candelaria. In: FERNÁNDEZ, A. (Comp.). Con letra de mujer: La crónica de las Hijas de María Auxiliadora en la Misión Nuestra Señora de la Candelaria, Tierra del Fuego. Buenos Aires: Didascalia, [1895-1916] 2014.

DEL TECHO, N. Historia de la provincia del Paraguay de la Compañía de Jesus. Versión del texto latino por Manuel Serrano y Sanz, con un prólogo de Blas Garay. Madrid: Uribe y Cía., [1673] 1897.

Diario de la Misión Nuestra Señora de La Candelaria (Inédito). Disponible en Museo Histórico y Natural Monseñor Fagnano (La Candelaria, Río Grande, Tierra del Fuego), [1896-1947]. 
GUEVARA, J. Historia de la conquista del Paraguay, Río de la Plata y Tucumán. Tomo Colección de obras, documentos y noticias inéditas o poco conocidas para servir a la historia física, política y literaria del Río de la Plata. Buenos Aires: S. Ostwald, [1764] 1882.

IL BOLLETINO SALESIANO. Disponible en: http://biesseonline.sdb.org/, [1878-1933], 2014.

LOZANO, P. Descripción Corográfica del Gran Chaco Gualamba. Tucumán: Instituto de Antropología, [1733] 1941.

LOZANO, P. Historia de la conquista de las provincias del Paraguay, Río de la Plata y Tucumán. Contiene la población de sus ciudades y progresos del dominio español en esta parte de la América meridional, tomo II. [1745] 2010

$\mathrm{MACHONI}$, A. Arte y vocabulario de la lengua lule, y tonocote compuestos con facultad de sus superiores por el Padre Antonio Machoni de Cerdeña. Madrid: Herederos de Juan García Infanzón,

http://www.filologiasarda.eu/files/documenti/pubblicazioni pdf/cfsmac cioni vocabolari/04edizione.pdf

OÑATE, P. de. Carta de Pedro de Oñate. En: RAVIGNANI, E. \& LEONHARDT, P. C. Cartas Anuas de la Provincia del Paraguay, Chile y Tucumán, de la Compañía de Jesús (1615-1637). In: Documentos para la historia argentina, tomo XX. Buenos Aires: Facultad de Filosofía y Letras, Instituto de Investigaciones históricas, p. 164-221, [1620] 1929.

OÑATE, P. de. Carta de Pedro de Oñate. In: RAVIGNANI, E. \& LEONHARDT, P. C. Cartas Anuas de la Provincia del Paraguay, Chile y Tucumán, de la Compañía de Jesús (1615-1637). Documentos para la historia argentina, tomo XX. Buenos Aires: Facultad de Filosofía y Letras, Instituto de Investigaciones históricas, p. 109-163, [1618] 1929.

VÁZQUEZ TRUJILLO, F. Carta de Francisco Vázquez Trujillo. In: RAVIGNANI, E. \& LEONHARDT, P. C. Cartas Anuas de la Provincia del Paraguay, Chile y Tucumán, de la Compañía de Jesús (16151637). Documentos para la historia argentina, tomo XX. Buenos Aires: Facultad de Filosofía y Letras, Instituto de Investigaciones históricas, p. 385-415, [1632] 1929.

ZURBANO, F. L. de. Anales de la provincia del Paraguay, desde el año de 35 hasta el de 37. In: RAVIGNANI, E. \& LEONHARDT, P. C. Cartas Anuas de la Provincia del Paraguay, Chile y Tucumán, de la Compañía de Jesús (1615-1637). Documentos para la historia argentina, tomo XX. Buenos Aires: Facultad de Filosofía y Letras, Instituto de Investigaciones históricas, p. 439-517, [1638] 1929. 